\title{
A Bayesian Abstract Economy with a Measure Space of Agents
}

\author{
Monica Patriche \\ Department of Probability, Statistics and Operations Research, Faculty of Mathematics and \\ Computer Science, University of Bucharest, Street Academiei 14, 010014 Bucharest, Romania \\ Correspondence should be addressed to Monica Patriche, monica.patriche@yahoo.com
}

Received 7 April 2009; Accepted 28 August 2009

Recommended by Nikolaos Papageorgiou

We define the model of an abstract economy with differential (asymmetric) information and a measure space of agents. We generalize N. C. Yannelis's result (2007), considering that each agent is characterised by a random preference correspondence instead of having a random utility function. We establish two different equilibrium existence results.

Copyright (C) 2009 Monica Patriche. This is an open access article distributed under the Creative Commons Attribution License, which permits unrestricted use, distribution, and reproduction in any medium, provided the original work is properly cited.

\section{Introduction}

We define the model of an abstract economy with asymmetric information and a measure set of agents, each of which is characterized by a private information set, an action (strategy) correspondence, a random constraint correspondence, and a random preference correspondence. The preference correspondences need not be representable by utility functions. The equilibrium concept is an extension of the deterministic equilibrium. We also present the model of Yannelis (see [1]) in which the agents maximize their expected utilities. Our model is a generalization of Yannelis's model.

A purpose of this paper is to prove the existence of equilibrium for an abstract economy with differential information and a measure space of agents. The assumptions on correspondences refer to upper semicontinuity and measurable graph. We use in this paper several results on the continuity and measurability of the set of integrable selections from a Banach-valued correspondences.

The model of an abstract economy with differential (asymmetric) information captures the meaning of trades under uncertainty. All economic activity in a society is made under conditions of uncertainty (incomplete information). The asymmetric information in the Arrow-Debreu model was introduced by Radner [2]. In his model each agent has his own private information set which is described by a partition of an exogenously given set of states 
of nature. The information partition of each agent generates a $\sigma$-algebra, and his net trades are measurable with respect to it (this $\sigma$-algebra). Thus, optimal choices reflect the private information of each agent.

The paper is organized as follows. In Section 2, some notational and terminological conventions are given. We also present, for the reader's convenience, some results on Bochner integration. In Section 3, Yannelis's expected utility model of differential information abstract economy and his main result in [1] are presented. Section 4 introduces our model, that is, the abstract economy with asymmetric information and a continuum of agents. Section 5 contains existence results for upper semicontinuous correspondences.

\section{Mathematical Preliminaries}

\subsection{Notation and Definition}

Throughout this paper, we will use the following notation:

(1) $\mathbb{R}_{++}$denotes the set of strictly positive reals,

(2) $\operatorname{co} A$ denotes the convex hull of the set $A$,

(3) $\overline{\mathrm{co}} A$ denotes the closed convex hull of the set $A$,

(4) $2^{A}$ denotes the set of all nonempty subsets of the set $A$,

(5) if $A \subset X$, where $X$ is a topological space, $\operatorname{cl} A$ denotes the closure of $A$.

For the reader's convenience, we review a few basic definitions and results from continuity and measurability of correspondences, Bochner integrable functions, and the integral of a correspondence.

Let $X$ and $Y$ be sets.

Definition 2.1. The graph of the correspondence $\Phi: X \rightarrow 2^{Y}$ is the set $G_{\Phi}=\{(x, y) \in X \times Y$ : $y \in \Phi(x)\}$.

Let $X, Y$ be topological spaces and let $\Phi: X \rightarrow 2^{Y}$ be a correspondence.

(1) $\Phi$ is said to be upper semicontinuous if for each $x \in X$ and each open set $V$ in $Y$ with $\Phi(x) \subset V$, there exists an open neighborhood $U$ of $x$ in $X$ such that $\Phi(y) \subset V$ for each $y \in U$.

(2) $\Phi$ is said to be lower semicontinuous if for each $x \in X$ and each open set $V$ in $Y$ with $\Phi(x) \cap V \neq \emptyset$, there exists an open neighborhood $U$ of $x$ in $X$ such that $\Phi(y) \cap V \neq \emptyset$ for each $y \in U$.

(3) $\Phi$ is said to have open lower sections if $\Phi^{-1}(y):=\{x \in X: y \in \Phi(x)\}$ is open in $X$ for each $y \in Y$.

Lemma 2.2 (see [3]). Let $X$ and $Y$ be two topological spaces and let $A$ be an open subset of $X$. Suppose that $\Phi_{1}: X \rightarrow 2^{Y}, \Phi_{2}: X \rightarrow 2^{Y}$ are upper semicontinuous such that $\Phi_{2}(x) \subset \Phi_{1}(x)$ for all $x \in A$. Then the correspondence $\Phi: X \rightarrow 2^{Y}$ defined by

$$
\Phi(x)= \begin{cases}\Phi_{1}(x), & \text { if } x \notin A, \\ \Phi_{2}(x), & \text { if } x \in A\end{cases}
$$

is also upper semicontinuous. 
Let now $(\Omega, \mathcal{F}, \mu)$ be a complete, finite measure space, and let $Y$ be a topological space.

Definition 2.3. (1) The correspondence $\Phi: \Omega \rightarrow 2^{Y}$ is said to have a measurable graph if $G_{\Phi} \in$ $\mathcal{F} \otimes \beta(Y)$, where $\beta(Y)$ denotes the Borel $\sigma$-algebra on $Y$ and $\otimes$ denotes the product $\sigma$-algebra.

(2) The correspondence $\Phi: \Omega \rightarrow 2^{Y}$ is said to be lower measurable if for every open subset $V$ of $Y$, the set $\{\omega \in \Omega: \Phi(\omega) \cap V \neq \emptyset\}$ is an element of $\mathcal{F}$.

Recall (see Debreu [4, page 359]) that if $\Phi: \Omega \rightarrow 2^{Y}$ has a measurable graph, then $\Phi$ is lower measurable. Furthermore, if $\Phi(\cdot)$ is closed valued and lower measurable, then $\Phi: \Omega \rightarrow 2^{\Upsilon}$ has a measurable graph.

Lemma 2.4 (see [5]). Let $\Phi_{n}: \Omega \rightarrow 2^{Y}, n=1,2, \ldots$ be a sequence of correspondences with measurable graphs. Then the correspondences $\bigcup_{n} \Phi_{n}, \cap_{n} \Phi_{n}$ and $Y \backslash \Phi_{n}$ have measurable graphs.

Let $(\Omega, \mathcal{F}, \mu)$ be a measure space and $Y$ be a Banach space.

It is known (see [6, Theorem 2, page 45]) that if $x: \Omega \rightarrow Y$ is a $\mu$-measurable function, then $x$ is Bochner integrable if only if $\int_{\Omega}\|x(\omega)\| d \mu(\omega)<\infty$.

It is denoted by $L_{1}(\mu, Y)$ the space of equivalence classes of $Y$-valued Bochner integrable functions $x: \Omega \rightarrow Y$ normed by $\|x\|=\int_{\Omega}\|x(\omega)\| d \mu(\omega)$.

Also it is known (see [6, page 50]) that $L_{1}(\mu, Y)$ is a Banach space.

We denote by $S_{\Phi}$ the set of all selections of the correspondence $\Phi: \Omega \rightarrow 2^{\gamma}$ that belong to the space $L_{1}(\mu, Y)$, that is,

$$
S_{\Phi}=\left\{x \in L_{1}(\mu, Y): x(\omega) \in \Phi(\omega) \mu \text {-a.e. }\right\}
$$

Definition 2.5 (see [7]). The integral of correspondence $\Phi: \Omega \rightarrow 2^{\gamma}$ is the set $\left\{\int_{\Omega} x(\omega) d \mu(\omega)\right.$ : $\left.x \in S_{\Phi}\right\}$.

We will denote the above set by $\int \Phi(\omega) d \omega$ or simply $\int \Phi$.

Definition 2.6. The correspondence $\Phi: \Omega \rightarrow 2^{\Upsilon}$ is said to be integrably bounded if there exists a map $h \in L_{1}(\mu, R)$ such that $\sup \{\|x\|: x \in \Phi(\omega)\} \leq h(\omega) \mu$-a.e.

Moreover, note that if $\Omega$ is a complete measure space, $Y$ is a separable Banach space and $\Phi: \Omega \rightarrow 2^{Y}$ is an integrably bounded, nonempty valued correspondence having a measurable graph; then by the Aumann measurable selection theorem we can conclude that $S_{\Phi}$ is nonempty and therefore $\int_{\Omega} \Phi(\omega) d \mu(\omega)$ is nonempty as well.

Let $X$ be a topological space and let $\Phi: \Omega \times X \rightarrow 2^{Y}$ be a nonempty valued correspondence.

Definition 2.7. A function $f: \Omega \times X \rightarrow Y$ is said to be a Carathéodory-type selection from $\Phi$ if $f(\omega, x) \in \Phi(\omega, x)$ for all $(\omega, x) \in \Omega \times X, f(\cdot, x)$ is measurable for all $x \in X$ and let $f(\omega, \cdot)$ is continuous for all $\omega \in \Omega$.

The results below have been used in the proof of our theorems. For more details and further references see the paper quoted.

Theorem 2.8 (Projection theorem). Let $(\Omega, \mathcal{F}, \mu)$ be a complete, finite measure space, and let $Y$ be a complete separable metric space. If $H$ belongs to $\mathcal{F} \otimes \beta(Y)$, its projection $\operatorname{Proj}_{\Omega}(H)$ belongs to $\mathcal{F}$. 
Theorem 2.9 (Aumann measurable selection theorem [2]). Let $(\Omega, \mathcal{F}, \mu)$ be a complete finite measure space, let $Y$ be a complete, separable metric space, and let $\Phi: \Omega \rightarrow 2^{Y}$ be a nonempty valued correspondence with a measurable graph, that is, $G_{\Phi} \in \mathcal{F} \otimes \beta(Y)$. Then there is a measurable function $f: \Omega \rightarrow Y$ such that $f(\omega) \in \Phi(\omega) \mu$-a.e.

Theorem 2.10 (Diestel's theorem [2, Theorem 3.1]). Let $(\Omega, \mathcal{F}, \mu)$ be a complete finite measure space, let $X$ be a separable Banach space, and let $\Phi: \Omega \rightarrow 2^{Y}$ be an integrably bounded, convex, weakly compact and nonempty valued correspondence. Then $S_{\Phi}=\left\{x \in L_{1}(\mu, Y): x(\omega) \in\right.$ $\Phi(\omega) \mu$-a.e. $\}$ is weakly compact in $L_{1}(\mu, Y)$.

Theorem 2.11 (Carathéodory-type selection theorem [5]). Let $(\Omega, \mathcal{F}, \mu)$ be a complete measure space, let $Z$ be a complete separable metric space, and let $Y$ be a separable Banach space. Let $X: \Omega \rightarrow$ $2^{Y}$ be a correspondence with a measurable graph, that is, $G_{X} \in \mathcal{F} \otimes \beta(Y)$ and let $\Phi: \Omega \times Z \rightarrow 2^{Y}$ be a convex valued correspondence (possibly empty) with a meaurable graph, that is, $G_{\Phi} \in \mathcal{F} \otimes \beta(Z) \otimes \beta(Y)$ where $\beta(Y)$ and $\beta(Z)$ are the Borel $\sigma$-algebras of $Y$ and $Z$, respectively.

Suppose that

(1) for each $\omega \in \Omega, \Phi(\omega, x) \subset X(\omega)$ for all $x \in Z$,

(2) for each $\omega \in \Omega, \Phi(\omega, \cdot)$ has open lower sections in $Z$; that is, for each $\omega \in \Omega$ and $y \in Y$, $\Phi^{-1}(\omega, y)=\{x \in Z: y \in \Phi(\omega, x)\}$ is open in $Z$,

(3) for each $(\omega, x) \in \Omega \times Z$, if $\Phi(\omega, x) \neq \emptyset$, then $\Phi(\omega, x)$ has a nonempty interior in $X(\omega)$.

Let $U=\{(\omega, x) \in \Omega \times Z: \Phi(\omega, x) \neq \emptyset\}$ and for each $x \in Z, U^{x}=\{\omega \in \Omega:(\omega, x) \in U\}$ and for each $\omega \in \Omega, U^{\omega}=\{x \in Z:(\omega, x) \in U\}$. Then for each $x \in Z, U^{x}$ is a measurable set in $\Omega$ and there exists a Caratheodory-type selection from $\Phi_{\mid U}$; that is, there exists a function $f: U \rightarrow Y$ such that $f(\omega, x) \in \Phi(\omega, x)$ for all $(\omega, x) \in U$, for each $x \in Z, f(\cdot, x)$ is measurable on $U^{x}$, and for each $\omega \in \Omega, f(\omega, \cdot)$ is continuous on $U^{\omega}$. Moreover, $f(\cdot, \cdot)$ is jointly measurable.

Theorem 2.12 (u.s.c. lifting theorem [2]). Let $Y$ be a separable space, let $(\Omega, \mathcal{F}, \mu)$ be a complete finite measure space, and let $X: \Omega \rightarrow 2^{Y}$ be an integrably bounded, nonempty, convex valued correspondence such that for all $\omega \in \Omega, X(\omega)$ is a weakly compact, convex subset of $Y$. Denote by $S_{X}$ the set $\left\{x \in L_{1}(\mu, Y): x(\omega) \in X(\omega) \mu\right.$-a.e. $\}$. Let $\Phi: \Omega \times S_{X} \rightarrow 2^{Y}$ be a nonempty, closed, convex valued correspondence such that $\Phi(\omega, x) \subset X(\omega)$ for all $(\omega, x) \in \Omega \times S_{X}^{1}$. Assume that for each fixed $x \in S_{X}, \Phi(\cdot, x)$ has a measurable graph and that for each fixed $\omega \in \Omega, \Phi(\omega, \cdot): S_{X} \rightarrow 2^{Y}$ is u.s.c. in the sense that the set $\left\{x \in S_{X}: \Phi(\omega, x) \subset V\right\}$ is weakly open in $S_{X}$ for every norm open subset $V$ of $Y$. Define the correspondence $\Psi: S_{X} \rightarrow 2^{S_{X}}$ by

$$
\Psi(x)=\left\{y \in S_{X}: y(\omega) \in \Phi(\omega, x) \mu \text {-a.e. }\right\}
$$

Then $\Psi$ is weakly u.s.c.; that is, the set $\left\{x \in S_{X}: \Psi(x) \subset V\right\}$ is weakly open in $S_{X}$ for every weakly open subset $V$ of $S_{X}$.

Theorem 2.13 (Measurability lifting theorem [8]). Let $Y$ and $E$ be separable Banach spaces, and let $(T, \tau, v)$ and $(\Omega, \mathcal{F}, \mu)$ be finite complete separable measure spaces. Let $\gamma: T \times \Omega \times E \rightarrow 2^{\gamma}$ be a nonempty valued correspondence. Suppose that for each $y \in E, \gamma(\cdot, \cdot, y)$ has a measurable graph. Define the correspondence $\psi: \Omega \times E \rightarrow 2^{L_{1}(\mu, Y)}$ by

$$
\psi(t, y)=\left\{x(t) \in L_{1}(\mu, Y): x(t, \omega) \in \gamma(t, \omega, y) \mu \text {-a.e. }\right\}
$$

Then for each $y \in E, \psi(\cdot, y)$ has a measurable graph. 


\section{A Bayesian Social Equilibrium Existence Theorem}

We present Yannelis's model [1] of an abstract economy with asymmetric information and a continuum of agents. In this model, there is assigned to each agent, in addition to his/her random utility function, a private information set, which is a measurable partition of the exogenously given probability measure space (which describes the states of nature of the world).

Let $(\Omega, \mathcal{F}, \mu)$ be a complete finite measure space, where $\Omega$ denotes the set of states of nature of the world and the $\sigma$-algebra $\mathcal{F}$ denotes the set of events. Let $Y$ be a separable Banach space whose dual has the RNP, denoting the commodity or strategy space.

Definition 3.1. A Bayesian abstract economy (or social system) with differential information and a measure space of agents $(T, \tau, v)$ is a set $G=\left\{\left(X, u, A, \mathcal{F}_{t}, q_{t}\right), t \in T\right\}$, where

(1) $X: T \times \Omega \rightarrow 2^{Y}$ is the random action (strategy) correspondence, where $X(t, \omega) \subset Y$ is interpreted as the strategy set of agent $t$ of the state of nature $\omega$;

(2) for each fixed $(t, \omega) \in T \times \Omega, u(t, \omega, \cdot, \cdot): L_{1}(v, Y) \times X(t, \omega) \rightarrow \mathbb{R}$ is the random utility function, where $u\left(t, \omega, x, x_{t}\right)$ is interpreted as the utility function of agent $t$, at the state of nature $\omega$, using his/her strategy $x_{t}$ and all other players use the joint strategy $x$;

(3) $A: T \times \Omega \times L_{1}(v, Y) \rightarrow 2^{Y}$ is the random constraint correspondence of agent $t$, where for all $(t, \omega, x) \in T \times \Omega \times L_{1}(v, Y), A(t, \omega, x) \subset X(t, \omega)$, and $A(t, \omega, x)$ is interpreted as the constraint of agent $t$, when the state is $\omega$ and other agents use the joint strategy $x$;

(4) $\mathcal{F}_{t}$ is a sub- $\sigma$-algebra of $\mathcal{F}$ which denotes the private information of agent $t$;

(5) $q_{t}: \Omega \rightarrow \mathbb{R}_{++}$is the prior of agent $t$, which is a Radon-Nikodym derivative such that $\int_{\Omega} q_{t}(\omega) d \mu(\omega)=1$.

Let $S_{X_{t}}^{1}=\left\{y(t) \in L_{1}(\mu, X): y(t, \cdot): \Omega \rightarrow Y\right.$ be $\mathscr{F}_{t}$-measurable and $y(t, \omega) \in$ $X(t, \omega) \mu$-a.e. $\}$. Notice that $S_{X_{t}}^{1}$ is the set of all Bocner integrable and $\mathscr{F}_{t}$-measurable selections from the random strategy of agent $t$. In essence this is the set, out of which agent $t$ will pick his/her optimal choices. In particular, an element $x_{t}$ in $S_{X_{t}}^{1}$ is called a strategy for agent $t$. The typical element of $S_{X_{t}}^{1}$ is denoted by $\tilde{x}_{t}$ and that of $X(t, \omega)$ by $x_{t}(\omega)$. Let $S_{X}^{1}=\{\tilde{x} \in$ $L_{1}\left(v, L_{1}(\mu, Y)\right): \tilde{x}(t, \cdot) \in S_{X_{t}}^{1} v$-a.e. $\}$. An element of $S_{X}^{1}$ will be a joint strategy profile.

It will be convenient to assume that $\Omega$ is a countable set and the $\sigma$-algebra $\mathcal{F}_{t}$ is generated by a countable partition $\Lambda$ of $\Omega$. For each $\omega \in \Omega$, let $E_{t}(\omega)$ in $\Lambda$ denote the smallest set in $\mathcal{F}_{t}$ containing $\omega$ and assume that, for each $t$,

$$
\int_{\omega^{\prime} \in E_{t}(\omega)} q_{t}\left(\omega^{\prime}\right) d \mu\left(\omega^{\prime}\right)>0
$$

Definition 3.2. For each $(t, \omega) \in T \times \Omega$, the interim expected utility of agent $t, U(t, \omega, \cdot, \cdot)$ : $S_{X}^{1} \times X(t, \omega) \rightarrow \mathbb{R}$ is defined as

$$
U\left(t, \omega, \tilde{x}, x_{t}\right)=\int_{\omega^{\prime} \in E_{t}(\omega)} u\left(t, \omega^{\prime}, \tilde{x}\left(\omega^{\prime}\right), x_{t}\left(\omega^{\prime}\right)\right) q_{t}\left(\omega^{\prime} \mid E_{t}(\omega)\right) d \mu\left(\omega^{\prime}\right)
$$


where

$$
q_{t}\left(\omega^{\prime} \mid E_{t}(\omega)\right)= \begin{cases}0, & \text { if } \omega^{\prime} \notin E_{t}(\omega) \\ \frac{q_{t}\left(\omega^{\prime}\right)}{\int_{\bar{\omega} \in E_{t}(\omega)} q_{t}(\bar{\omega}) d \mu(\bar{\omega})}, & \text { if } \omega^{\prime} \in E_{t}(\omega) .\end{cases}
$$

Definition 3.3. A social equilibrium for $G$ is a strategy profile $\tilde{x}^{*} \in S_{X}^{1}$ such that $v$-a.e.

(i) $\tilde{x}^{*}(t, \omega) \in A\left(t, \omega, \tilde{x}^{*}\right) \mu$-a.e.,

(ii) $U\left(t, \omega, \tilde{x}, \tilde{x}^{*}(t, \omega)\right)=\max _{y \in A\left(t, \omega, \tilde{x}^{*}\right)} U(t, \omega, \tilde{x}, y) \mu$-a.e.

The following theorem is the main result of Yannelis in [1].

Theorem 3.4. Let $G$ be a social system with asymmetric information satisfying (A.1)-(A.4). Then there exists a social equilibrium for $G$.

One has the following assumptions:

(A.1)

(a) $X: T \times \Omega \rightarrow 2^{Y}$ is a nonempty, convex, compact valued, and integrably bounded correspondence,

(b) for each $t \in T, X(t, \cdot): \Omega \rightarrow 2^{Y}$ has an $\mathcal{F}_{t}$ measurable graph, that is, for every open subset $V$ of $Y$, the set $G_{X}(t, \cdot) \in \mathcal{F}_{t} \times \beta(Y)$.

(A.2)

(a) for each $(t, \omega) \in T \times \Omega, u(t, \omega, \cdot, \cdot): L_{1}(v, Y) \times X(t, \omega) \rightarrow \mathbb{R}$ is continuous where $L_{1}(v, Y)$ is endowed with the weak topology and $X(t, \omega)$ with the norm topology,

(b) for each fixed $(x, y) \in L_{1}(v, Y) \times Y, u(\cdot, \cdot, x, y): T \times \Omega \rightarrow \mathbb{R}$ is a measurable function,

(c) for each $(t, \omega, x) \in T \times \Omega \times L_{1}(v, Y), u(t, \omega, x, \cdot): X(t, \omega) \rightarrow \mathbb{R}$ is concave,

(d) for each $t \in T, u(t, \cdot, \cdot, \cdot)$ is integrably bounded.

(a) $A: T \times \Omega \times L_{1}(v, Y) \rightarrow 2^{Y}$ has a measurable graph,

(b) for each $(t, \omega) \in T \times \Omega, A(t, \omega, \cdot): L_{1}(v, Y) \times \rightarrow 2^{Y}$ is a continuous correspondence with closed, convex, and nonempty values.

(A.4) The correspondence $t \rightarrow S_{X_{t}}^{1}$ has a measurable graph.

Remark 3.5. This theorem and its proof remain unchanged if the random constraint correspondence is defined as $A_{i}: \Omega \times L_{X} \rightarrow 2^{Y}$.

\section{The Model}

We will study the next model of the abstract economy.

Let $(\Omega, F, \mu)$ be a complete finite measure space, where $\Omega$ denotes the set of states of nature of the world and the $\sigma$-algebra $\mathcal{F}$ denotes the set of events. Let $Y$ be a separable Banach space whose dual has the RNP, denoting the commodity or strategy space. 
Definition 4.1. A Bayesian abstract economy (or social system) with differential information and a measure space of agents $(T, \tau, v)$ is a set $G=\left\{\left(X, F_{t}, A, P\right), t \in T\right\}$, where

(1) $X: T \times \Omega \rightarrow 2^{Y}$ is the random action (strategy) correspondence, where, $X(t, \omega) \subset Y$ is interpreted as the strategy set of agent $t$ of the state of nature $\omega$;

(2) $\mathcal{F}_{t}$ is a sub- $\sigma$-algebra of $\mathcal{F}$ which denotes the private information of agent $t$;

(3) for each $t \in T, A(t, \cdot, \cdot): \Omega \times S_{X}^{1} \rightarrow 2^{Y}$ is the random constraint correspondence of agent $t$, where for all $(t, \omega, x) \in T \times \Omega \times S_{X}^{1}, A(t, \omega, x) \subset X(t, \omega)$;

(4) for each $t \in T, P(t, \cdot, \cdot): \Omega \times S_{X}^{1} \rightarrow 2^{Y}$ is the random preference correspondence of agent $t$, where for all $(t, \omega, x) \in T \times \Omega \times S_{X^{\prime}}^{1} P(t, \omega, x) \subset X(t, \omega)$.

Definition 4.2. A Bayesian equilibrium for $G$ is a strategy profile $\tilde{x}^{*} \in S_{X}^{1}$ such that for $v$-a.e.

(i) $\tilde{x}^{*}(t, \omega) \in A\left(t, \omega, \tilde{x}^{*}\right) \mu$-a.e.,

(ii) $A\left(t, \omega, \tilde{x}^{*}\right) \cap P\left(t, \omega, \tilde{x}^{*}\right)=\emptyset \mu$-a.e.

Remark 4.3. This model of abstract economy is a generalization of Yannelis's model presented in Section 3, since for intern expected utilities $U$ we can define the correspondence $P: T \times \Omega \times$ $S_{X}^{1} \rightarrow 2^{Y}$ by $P(t, \omega, x)=\{y \in Y: U(t, \omega, x(t, \omega), y)>U(t, \omega, x(t, \omega) \mu$-a.e. $\}$.

\section{Bayesian Equilibrium Existence Theorems}

Now we establish an equilibrium existence theorem for Bayesian abstract economies with a measure space of agents and with upper semicontinuous correspondences. Our theorem generalizes Theorem 1 in [1].

Theorem 5.1. Let $(T, \tau, v)$ be a measure space of agents and let $G=\left\{\left(X, \mathcal{F}_{t}, A, P\right), t \in T\right\}$ be a Bayesian abstract economy satisfying (A.1)-(A.5). Then there exists a Bayesian equilibrium for G.

(a) $X: T \times \Omega \rightarrow 2^{Y}$ is a nonempty, convex, weakly compact-valued, and integrably bounded correspondence,

(b) for each fixed $t \in T, X(t, \cdot)$ has an $\mathcal{F}_{t}$-measurable graph, that is, for every open subset $V$ of $Y$, the set $G_{X(t,)} \in F_{t} \times \beta(Y)$.

(a) $A: T \times \Omega \times S_{X}^{1} \rightarrow 2^{Y}$ has a measurable graph,

(b) for each $(t, \omega) \in T \times \Omega, A(t, \omega, \cdot): S_{X}^{1} \rightarrow 2^{Y}$ is an upper semicontinuous correspondence with closed, convex and nonempty values. 
(A.3)

(a) $P: T \times \Omega \times S_{X}^{1} \rightarrow 2^{Y}$ has a measurable graph,

(b) for each $(t, \omega) \in T \times \Omega, P(t, \omega, \cdot): S_{X}^{1} \rightarrow 2^{\gamma}$ is an upper semicontinuous correspondence with closed, convex, and nonempty values.

(A.4) The correspondence $t \rightarrow S_{X_{t}}^{1}$ has a measurable graph.

(a) for each $\omega \in \Omega$, for each $x(t) \in S_{X^{\prime}}^{1} x(t, \omega) \notin A(t, \omega, x) \cap P(t, \omega, x)$,

(b) the set $U^{(t, \omega)}=\left\{x \in S_{X}^{1}: A(t, \omega, x) \cap P(t, \omega, x) \neq \emptyset\right\}$ is weakly open in $S_{X}^{1}$.

Proof. Define $\Phi: T \times \Omega \times S_{X}^{1} \rightarrow 2^{Y}$ by $\Phi(t, \omega, x)=A(t, \omega, x) \cap P(t, \omega, x)$. We will prove first that $S_{X}^{1}$ is nonempty, convex, weakly compact.

Since $(\Omega, \mathscr{F}, \mu)$ is a complete finite measure space, $Y$ is a separable Banach space and $X(t, \cdot): \Omega \rightarrow 2^{Y}$ has measurable graph, and by Aumann's selection theorem it follows that there exists a function $f(t, \cdot): \Omega \rightarrow Y$ such that $f(t, \omega) \in X(t, \omega) \mu$-a.e. Since $X(t, \cdot)$ is integrably bounded, we have that $f(t, \cdot) \in L_{1}(\mu, Y)$, hence $S_{X_{t}}$ is nonempty, and $S_{X}=\prod_{t \in T} S_{X_{t}}$ is nonempty. $S_{X_{t}}^{1}$ is convex and $S_{X}^{1}$ is also convex. Since $X(t, \cdot): \Omega \rightarrow 2^{Y}$ is integrably bounded and has convex weakly compact values, by Diestel's Theorem it follows that $S_{X_{t}}^{1}$ is a weakly compact subset of $L_{1}(\mu, Y)$ and so is $S_{X}^{1}$. We have that $S_{X}^{1}$ is a metrizable set as being a weakly compact subset of the separable Banach space $L_{1}\left(v, L_{1}(\mu, Y)\right.$ ) (Dunford-Schwartz [9, page 434]).

Since all the values of the correspondence $A$ are contained in the compact set $X(\cdot, \cdot)$ and $A$ is closed and convex valued (hence weakly closed), it follows that $A$ is weakly compact valued.

Then $\Phi$ is convex valued and for each $(t, \omega) \in T \times \Omega, \Phi(t, \omega, \cdot)$ is upper semicontinuous. We have that $\Phi(\cdot, \cdot, x)$ has a measurable graph for each $x \in S_{X}^{1}$. Let $U=\left\{(t, \omega, x) \in \Omega \times S_{X}^{1}\right.$ : $\Phi(t, \omega, x) \neq \emptyset\}$. For each $x \in S_{X}^{1}$, let $U^{x}=\{(t, \omega) \in T \times \Omega: \Phi(t, \omega, x) \neq \emptyset\}$ and for each $\omega \in \Omega$, let $U^{(t, \omega)}=\left\{x \in S_{X}^{1}: \Phi(t, \omega, x) \neq \emptyset\right\}$. Define $G: T \times \Omega \times S_{X}^{1} \rightarrow 2^{\gamma}$ by

$$
G(t, \omega, x)= \begin{cases}\Phi(t, \omega, x), & \text { if }(t, \omega, x) \in U, \\ A(t, \omega, x), & \text { if }(t, \omega, x) \notin U .\end{cases}
$$

For each $x \in S_{X}^{1}$, the correspondence $G(\cdot, \cdot, x)$ has a measurable graph.

By assumption (A5) (b), the set $U^{(t, \omega)}=\left\{x \in S_{X}^{1}: A(t, \omega, x) \cap P(t, \omega, x) \neq \emptyset\right\}$ is weakly open in $S_{X}^{1}$. For each $(t, \omega) \in T \times \Omega, G(t, \omega, \cdot): S_{X}^{1} \rightarrow 2^{Y}$ is upper semicontinuous.

Let $V$ be weakly open in $S_{X}^{1}$ and $x \in S_{X}^{1}$ :

$$
\begin{aligned}
W & =\left\{x \in S_{X}^{1}: G(t, \omega, x) \subset V\right\} \\
& =\left\{x \in U^{(t, \omega)}: G(t, \omega, x) \subset V\right\} \cup\left\{x \in S_{X}^{1} \backslash U^{(t, \omega)}: G(t, \omega, x) \subset V\right\} \\
& =\left\{x \in U^{(t, \omega)}: \Phi(t, \omega, x) \subset V\right\} \cup\left\{x \in S_{X}^{1}: G(t, \omega, x) \subset V\right\} .
\end{aligned}
$$


$W$ is an open set, because $U^{(t, \omega)}$ is open, $\Phi(t, \omega, \cdot)$ is an upper semicontinuous map on $U^{(t, \omega)}$, and the set $\left\{x \in S_{X}^{1}: G(t, \omega, x) \subset V\right\}$ is open since $G(t, \omega, \cdot)$ is u.s.c. Moreover, $G$ is convex and nonempty valued.

Define the correspondence $\varphi: T \times S_{X}^{1} \rightarrow 2^{L_{1}(\mu, Y)}$ by $\varphi(t, \tilde{x})=\left\{\tilde{y}(t) \in L_{1}(\mu, Y): \widetilde{y}(t, \omega) \in\right.$ $G(t, \omega, \tilde{x}) \mu$-a.e. $\} \cap S_{X_{t}}^{1}$.

By the measurability lifting theorem (Theorem 2.13), the correspondence $t \rightarrow\{\tilde{y}(t) \in$ $L_{1}(\mu, Y): \tilde{y}(t, \omega) \in G(t, \omega, \tilde{x}) \mu$-a.e. $\}$ has a measurable graph and so does $t \rightarrow S_{X_{t}}^{1}$ by (A.4). Thus, for each fixed $\tilde{x} \in S_{X}^{1}, \varphi(\cdot, \tilde{x})$ has a measurable graph.

Since for each fixed $\tilde{x} \in S_{X}^{1}, G(\cdot, \cdot, \tilde{x})$ has a measurable graph and it is nonempty valued, then by the Aumann measurable selection theorem, it admits a measurable selection and we can conclude that $\varphi$ is nonempty valued. It follows by the u.s.c. lifting theorem that for each fixed $t, \varphi(t, \cdot)$ is weakly u.s.c.

Define $G^{\prime}: S_{X}^{1} \rightarrow 2^{S_{X}^{1}}$, by $G^{\prime}(\tilde{x})=\left\{\tilde{y} \in S_{X}^{1}: \tilde{y}(t) \in \varphi(t, \tilde{x})\right.$ v-a.e. $\}$.

Another application of the u.s.c. lifting theorem enables us to conclude that $G^{\prime}$ is a weakly u.s.c. correspondence which is obviously convex valued (since $\varphi$ is convex valued) and also nonempty valued (recall once more the Aumann measurable selection theorem and notice that the set $S_{X}^{1}$ is metrizable).

$G^{\prime}$ is an upper semicontinuous correspondence and has also nonempty convex closed values.

By Fan-Glicksberg's fixed-point theorem in [5], there exists $\tilde{x}^{*} \in S_{X}^{1}$ such that $\tilde{x}^{*} \in$ $G^{\prime}\left(\tilde{x}^{*}\right)$. It follows that $\tilde{x}^{*} \in S_{X}^{1}$ and $\tilde{x}^{*}(t) \in \varphi\left(t, \tilde{x}^{*}\right) \mathcal{v}$-a.e. Thus, we have that $\tilde{x}^{*} \in S_{X}^{1}$ and $\tilde{x}^{*}(t, \omega) \in G\left(t, \omega, \tilde{x}^{*}\right) \mu$-a.e., $v$-a.e.

By assumption (A.4)(a), it follows that $\tilde{x}^{*}(t, \omega) \notin(A \cap P)\left(t, \omega, \tilde{x}^{*}\right)$, then we have that $\tilde{x}^{*} \notin U$ and $\tilde{x}^{*}(t, \omega) \in A\left(t, \omega, \tilde{x}^{*}\right)$.

Therefore, for $v$-a.e.

(1) $\tilde{x}^{*}(t, \omega) \in A\left(t, \omega, \tilde{x}^{*}\right) \mu$-a.e.,

(2) $A\left(t, \omega, \tilde{x}^{*}\right) \cap P\left(t, \omega, \tilde{x}^{*}\right)=\emptyset \mu$-a.e.

If there exists a selector $F$ for $A \cap P$ such that it has measurable graph and it is weakly upper semicontinuous in the third argument, we obtain the following theorem.

Theorem 5.2. Let $(T, \tau, v)$ be a measure space of agents and let $G=\left\{\left(X, \mathcal{F}_{t}, A, P\right), t \in T\right\}$ be a Bayesian abstract economy satisfying (A.1)-(A.5). Then there exists a Bayesian equilibrium for $G$.

(A.1)

(a) $X: T \times \Omega \rightarrow 2^{Y}$ is a nonempty, convex, weakly compact-valued, and integrably bounded correspondence,

(b) for each fixed $t \in T, X(t, \cdot)$ has an $\mathcal{F}_{t}$-measurable graph, that is, for every open subset $V$ of $Y$, the set $G_{X}(t, \cdot) \in \mathcal{F}_{t} \times \beta(Y)$.

(a) $A: T \times \Omega \times S_{X}^{1} \rightarrow 2^{Y}$ has a measurable graph,

(b) for each $(t, \omega) \in T \times \Omega, A(t, \omega, \cdot): S_{X}^{1} \rightarrow 2^{Y}$ is an upper semicontinuous correspondence with closed convex and nonempty values. 
(A.3)

(a) $P: T \times \Omega \times S_{X}^{1} \rightarrow 2^{Y}$ has a measurable graph,

(b) for each $(t, \omega) \in T \times \Omega, P(t, \omega, \cdot): S_{X}^{1} \rightarrow 2^{Y}$ is a upper semicontinuous correspondence with closed, convex, and nonempty values.

(A.4)

(a) for each $(t, \omega) \in T \times \Omega$, the set $U^{(t, \omega)}=\left\{x \in S_{X}^{1}: A(t, \omega, x) \cap P(\omega, x)=\emptyset \mu\right.$-a.e. $\}$ is open in $S_{X^{\prime}}^{1}$

(b) for each $\omega \in \Omega$, for each $x \in U, x(t, \omega) \notin A(t, \omega, x) \cap P(t, \omega, x)$.

(a) there exists a selector $F: S_{X}^{1} \rightarrow 2^{Y}$ for $A \cap P: S_{X}^{1} \rightarrow 2^{Y}$ such that for each $x \in$ $S_{X^{\prime}}^{1}, F(\cdot, \cdot, x)$ has measurable graph and for each $(t, \omega) \in T \times \Omega, F(t, \omega, \cdot): U^{(t, \omega)} \rightarrow 2^{Y}$ is weakly upper semicontinuous with closed and convex values.

Proof. Define $G: T \times \Omega \times S_{X}^{1} \rightarrow 2^{\gamma}$ by

$$
G(t, \omega, x)= \begin{cases}F(t, \omega, x) & \text { if }(t, \omega, x) \in U, \\ A(t, \omega, x) & \text { if }(t, \omega, x) \notin U .\end{cases}
$$

For each $x \in S_{X}^{1}$, the correspondence $G(\cdot, \cdot, x)$ has a measurable graph.

By assumption (A4)(b), the set $U^{(t, \omega)}=\left\{x \in S_{X}^{1}: A(t, \omega, x) \cap P(t, \omega, x) \neq \emptyset\right\}$ is weakly open in $S_{X}^{1}$. For each $(t, \omega) \in T \times \Omega, G(t, \omega, \cdot): S_{X}^{1} \rightarrow 2^{Y}$ is upper semicontinuous.

Let $V$ be weakly open in $S_{X}^{1}$ and $x \in S_{X}^{1}$ :

$$
\begin{aligned}
W & =\left\{x \in S_{X}^{1}: G(t, \omega, x) \subset V\right\} \\
& =\left\{x \in U^{(t, \omega)}: G(t, \omega, x) \subset V\right\} \cup\left\{x \in S_{X}^{1} \backslash U^{(t, \omega)}: G(t, \omega, x) \subset V\right\} \\
& =\left\{x \in U^{(t, \omega)}: F(t, \omega, x) \subset V\right\} \cup\left\{x \in S_{X}^{1}: G(t, \omega, x) \subset V\right\} .
\end{aligned}
$$

$W$ is an open set, because $U^{(t, \omega)}$ is open, $\Phi(t, \omega, \cdot)$ is a upper semicontinuous map on $U^{(t, \omega)}$, and the set $\left\{x \in S_{X}^{1}: G(t, \omega, x) \subset V\right\}$ is open since $G(t, \omega, \cdot)$ is u.s.c. Moreover, $G$ is convex and nonempty valued.

Define the correspondence $\varphi: T \times S_{X}^{1} \rightarrow 2^{L_{1}(\mu, Y)}$ by $\varphi(t, \tilde{x})=\left\{\tilde{y}(t) \in L_{1}(\mu, Y): \tilde{y}(t, \omega) \in\right.$ $G(t, \omega, \tilde{x}) \mu$-a.e. $\} \cap S_{X_{t}}^{1}$.

By the measurability lifting theorem (Theorem 2.13), the correspondence $t \rightarrow\{\tilde{y}(t) \in$ $L_{1}(\mu, Y): \tilde{y}(t, \omega) \in G(t, \omega, \tilde{x}) \mu$-a.e. $\}$ has a measurable graph and so does $t \rightarrow S_{X_{t}}^{1}$ by (A.4). Thus, for each fixed $\tilde{x} \in S_{X}^{1}, \varphi(\cdot, \tilde{x})$ has a measurable graph.

Since for each fixed $\tilde{x} \in S_{X}^{1}, G(\cdot, \cdot, \tilde{x})$ has a measurable graph and it is nonempty valued, then by the Aumann measurable selection theorem, it admits a measurable selection and we can conclude that $\varphi$ is nonempty valued. It follows by the u.s.c. lifting theorem that for each fixed $t, \varphi(t, \cdot)$ is weakly u.s.c.

Define $G^{\prime}: S_{X}^{1} \rightarrow 2^{S_{X}^{1}}$, by $G^{\prime}(\tilde{x})=\left\{\tilde{y} \in S_{X}^{1}: \tilde{y}(t) \in \varphi(t, \tilde{x}) \mathcal{v}\right.$-a.e. $\}$. 
Another application of the u.s.c. lifting Theorem enables us to conclude that $G^{\prime}$ is a weakly u.s.c. correspondence which is obviously convex valued (since $\varphi$ is convex valued) and also nonempty valued (recall once more the Aumann measurable selection theorem and notice that the set $S_{X}^{1}$ is metrizable). values.

$G^{\prime}$ is an upper semicontinuous correspondence and has also nonempty convex closed

By Fan-Glicksberg's fixed-point theorem in [5], there exists $\tilde{x}^{*} \in S_{X}^{1}$ such that $\tilde{x}^{*} \in$ $G^{\prime}\left(\tilde{x}^{*}\right)$. It follows that $\tilde{x}^{*} \in S_{X}^{1}$ and $\tilde{x}^{*}(t) \in \varphi\left(t, \tilde{x}^{*}\right) \mathcal{v}$-a.e. Thus, we have that $\tilde{x}^{*} \in S_{X}^{1}$ and $\tilde{x}^{*}(t, \omega) \in G\left(t, \omega, \tilde{x}^{*}\right) \mu$-a.e., $v$-a.e.

By assumption (A.4)(a), it follows that $\tilde{x}^{*}(t, \omega) \notin(A \cap P)\left(t, \omega, \tilde{x}^{*}\right)$, then we have that $\tilde{x}^{*} \notin U$ and $\tilde{x}^{*}(t, \omega) \in A\left(t, \omega, \tilde{x}^{*}\right)$.

Therefore, for $v$-a.e.

(1) $\tilde{x}^{*}(t, \omega) \in A\left(t, \omega, \tilde{x}^{*}\right) \mu$-a.e.,

(2) $A\left(t, \omega, \tilde{x}^{*}\right) \cap P\left(t, \omega, \tilde{x}^{*}\right)=\emptyset \mu$-a.e.

\section{References}

[1] N. C. Yannelis, "Debreu's social equilibrium theorem with asymmetric information and a continuum of agents," Economic Theory, vol. 38, no. 2, pp. 419-432, 2007.

[2] R. Radner, "Competitive equilibrium under uncertainty," Econometrica, vol. 36, pp. 31-58, 1968.

[3] N. C. Yannelis, "Set-valued function of two variables in economic theory," in Equilibrium Theory in Infinite Dimensional Spaces, M. A. Khan and N. C. Yannelis, Eds., Studies in Economic Theory, Springer, Berlin, Germany, 1991.

[4] G. Debreu, "A social equilibrium existence theorem," Proceedings of the National Academy of Sciences of the United States of America, vol. 38, pp. 886-893, 1952.

[5] K. Fan, "Fixed-point and minimax theorems in locally convex topological linear spaces," Proceedings of the National Academy of Sciences of the United States of America, vol. 38, no. 2, pp. 121-126, 1952.

[6] G. Debreu, "Integration of correspondences," in Proceedings of the 5th Berkeley Symposium on Mathematical Statistics and Probability, vol. 2, pp. 351-372, University of California Press, 1966.

[7] R. J. Aumann, "Integrals of set-valued functions," Journal of Mathematical Analysis and Applications, vol. 12, pp. 1-12, 1965.

[8] E. J. Balder and N. C. Yannelis, "Equilibrium in random and Bayesian games with a continuum of players," in Equilibrium Theory in Infinite Dimensional Spaces, M. A. Khan and N. C. Yannelis, Eds., Springer, New York, NY, USA, 1991.

[9] N. Dunford and J. T. Schwartz, Linear Operators, Vol. I, Wiley-Interscience, New York, NY, USA, 1958. 


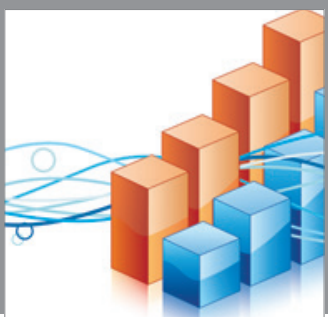

Advances in

Operations Research

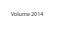

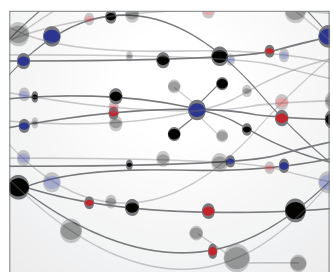

\section{The Scientific} World Journal
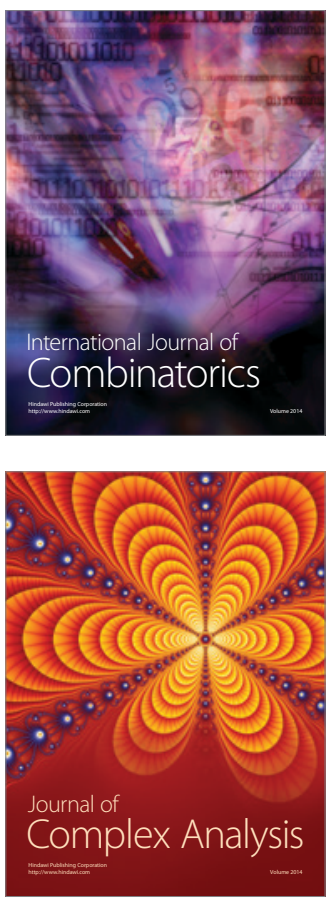

International Journal of

Mathematics and

Mathematical

Sciences
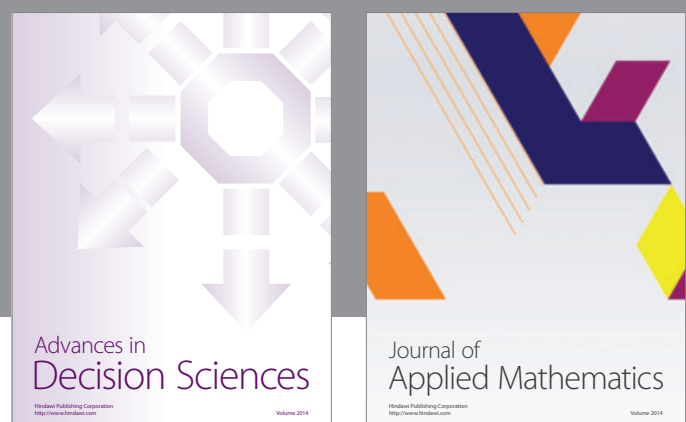

Journal of

Applied Mathematics
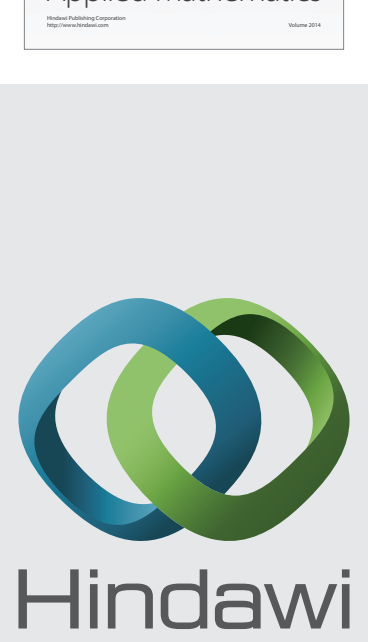

Submit your manuscripts at http://www.hindawi.com
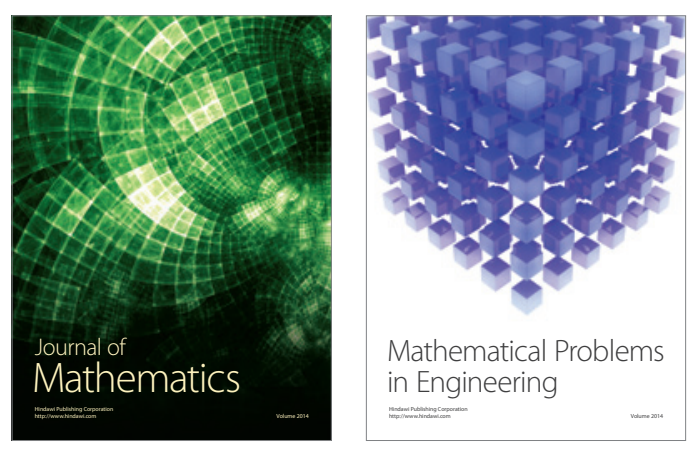

Mathematical Problems in Engineering
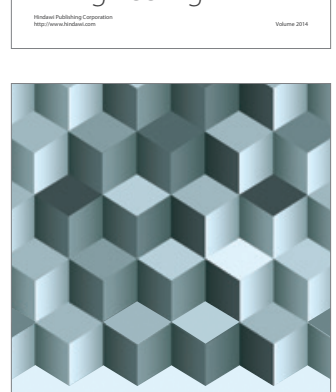

Journal of

Function Spaces
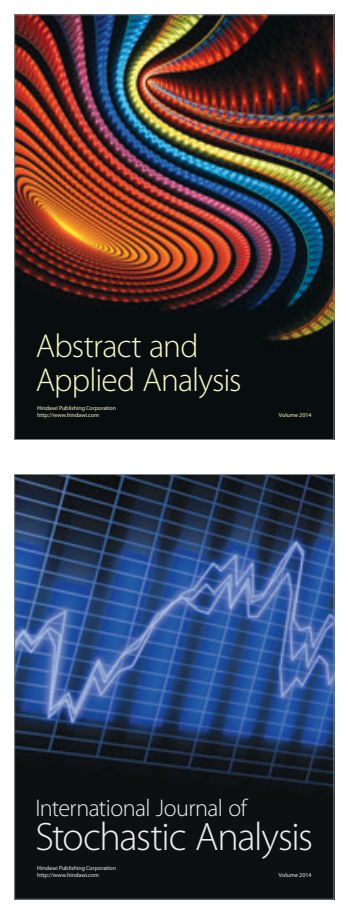

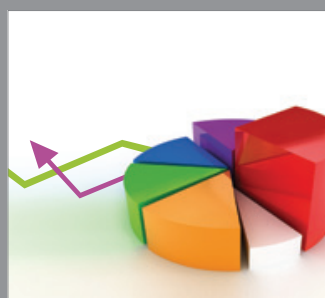

ournal of

Probability and Statistics

Promensencen
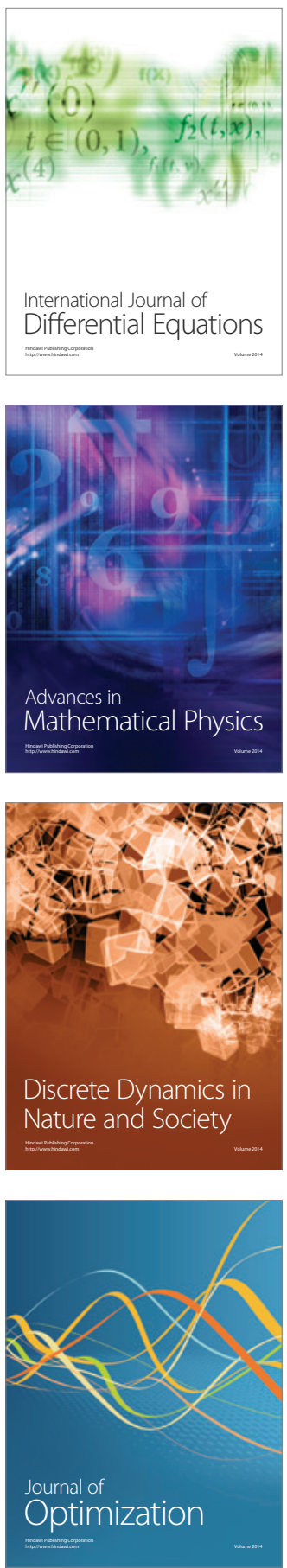